Revista de Metalurgia 50(1)

Enero-Marzo 2014, e007

ISSN-L: 0034-8570

doi: http://dx.doi.org/10.3989/revmetalm.007

\title{
Caracterización dinámica de los cambios microestructurales en barras de aceros dúplex SAF 2205 utilizando la dimensión de información
}

\author{
Edda Rodríguez $^{\mathrm{a}, \varpi}$, Kleydis Suárez $^{\mathrm{b}}$, Luis Amorer ${ }^{\mathrm{c}}$, Jesús Silva ${ }^{\mathrm{b}}$ \\ ${ }^{a}$ Universidad Simón Bolívar, Dpto. de Ciencia de los Materiales, Apartado 89000, Caracas 1080, Venezuela \\ ${ }^{b}$ Universidad Simón Bolívar, Dpto. de Tecnología Industrial, Apartado 89000,vCaracas 1080, Venezuela \\ 'Universidad Central de Venezuela, Facultad de Ingeniería, Dpto. de Física Aplicada, Caracas 1050, Venezuela \\ Autor para la correspondencia: eprato@usb.ve
}

Enviado: 29 Enero 2013; Aceptado: 23 Agosto 2013

\begin{abstract}
RESUMEN: En el presente estudio se evalúa el comportamiento dinámico de las señales ultrasónicas de muestras de acero dúplex SAF 2205 (UNS31803), sometidos a tratamientos de envejecimiento a 875 y $950{ }^{\circ} \mathrm{C}$. En el primer tratamiento se promueve la precipitación de la fase sigma y en el segundo su disolución. Los resultados obtenidos por microscopía óptica indican que la cantidad relativa de la fase sigma aumenta progresivamente con el tiempo de envejecimiento a $875^{\circ} \mathrm{C}$, observándose la transformación de la fase ferrita en fase sigma y en austenita secundaria. Por otra parte, a medida que se aumenta el tiempo de tratamiento a $950{ }^{\circ} \mathrm{C}$, se observa una reducción del porcentaje de fase sigma y un aumento de la fase ferrítica. El estudio propone evaluar en el ámbito de sistemas dinámicos la influencia de las fases microestructurales en el comportamiento de las señales y su dependencia con las pérdidas de energía, que ocurren en el sistema por efecto de los tratamientos térmicos, utilizando la teoría del caos determinista. Para caracterizarlos se emplea la representación del espacio de fases dinámicas y el cálculo de la dimensión de información. Los resultados obtenidos muestran una mayor correlación entre la dimensión de información y el coeficiente de atenuación, en comparación con las fracciones de fase. No obstante, la eficiencia de este tipo de estudio depende de la frecuencia seleccionada durante la evaluación del material.
\end{abstract}

PALABRAS CLAVE: Acero inoxidable SAF 2205; Caos determinístico; Dimensión de información; Espacio de fases: Inspección ultrasónica

Citation / Cómo citar este artículo: Rodríguez, E., Suárez, K., Amorer, L., Silva, J. (2014) "Caracterización dinámica de los cambios microestructurales en barras de aceros dúplex SAF 2205 utilizando la dimensión de información". Rev. Metal. 50(1): e007. doi: http://dx.doi.org/10.3989/revmetalm.007.

SUMMARY: Dynamics characterization of microstructural changes in bars of duplex steels SAF 2205 using the information dimension. This study evaluates the ultrasonic signals behavior of a duplex stainless steel SAF 2205 (UNS S31803) after aging treatments at 875 and $950{ }^{\circ} \mathrm{C}$. The first treatment promotes the precipitation of the sigma phase and the second, its dissolution. The microstructural evaluation was carried out by optical microscopy. The results showed an increase of the sigma phase fraction and the secondary austenite with the time of aging treatment at $875{ }^{\circ} \mathrm{C}$. On the other hand, increasing the time of treatment at $950{ }^{\circ} \mathrm{C}$ promotes a reduction of the sigma phase and the corresponding increase of the ferrite phase fraction. The study proposed to evaluate in the dynamical system field the influence of microstructural phases in the signals behavior and their dependence on the energy losses occurring in system during heat treatment, by deterministic chaos theory. To accomplish characterization, algorithm employs the representation of dynamic phase space and the information dimension estimate. The results show a higher correlation between the information dimension and the attenuation coefficient in comparison with the phase fractions. However, the efficiency of this type of study will depend on the frequency selected for the evaluation of the material.

KEYWORDS: Deterministic chaos; Dimension of information; Duplex stainless steel SAF 220; Dynamic phase space Ultrasonic testing

Copyright: $(\mathrm{C} 2014$ CSIC. This is an open-access article distributed under the terms of the Creative Commons AttributionNon Commercial (by-nc) Spain 3.0 License. 


\section{INTRODUCCIÓN}

La denominación dúplex se aplica a un tipo de acero inoxidable, cuya composición química ha sido ajustada para lograr una estructura cristalina mixta, de aproximadamente cantidades iguales de ferrita $(\alpha)$ y de austenita $(\gamma)$. Estos aceros contienen cromo, molibdeno y suficiente cantidad de estabilizadores de la $\gamma$, como lo son el níquel y el nitrógeno, para lograr el balance entre estas dos fases (Gunn, 1987). Aparte de las fases $\alpha$ y $\gamma$, una gran variedad de fases secundarias no deseadas suelen formarse en el intervalo de temperaturas entre 300 y $1000{ }^{\circ} \mathrm{C}$. Esto es esencialmente una consecuencia de la inestabilidad de la $\alpha$. Se han observado las siguientes fases: fase sigma $(\sigma), C r_{2} N$, austenita secundaria $\left(\gamma_{2}\right)$, fase $\chi$, fase $R$, fase $\pi, M_{7} C_{3}, M_{23} C_{6}, C u$, y fase $\tau$ (Gunn, 1987). La precipitación de la fase $\sigma$ es una de las que merece mayor atención por cuanto la presencia de pequeñas cantidades en el acero afecta drásticamente las propiedades mecánicas característica de estos aceros.

La fase $\sigma$ es una fase intermetálica no magnética de estructura tetragonal cuya composición se basa principalmente en los elementos hierro y cromo. En los aceros inoxidables dúplex, la precipitación de la fase $\sigma$ es observada en un intervalo de temperaturas entre 600 y $1000{ }^{\circ} \mathrm{C}$. La precipitación de la fase $\sigma$ ocurre principalmente a través de la descomposición eutectoide de $\alpha$ en $\sigma$ y en $\gamma_{2}$. Esta reacción comienza en la interfase $\alpha / \gamma$, donde la descomposición de $\alpha$ ocurre por la alta velocidad de difusión de los elementos que conforman la fase $\sigma$, como el cromo y el molibdeno, motivada por la similitud de la estructura tetragonal de la fase $\sigma$ y la estructura BCC de la $\alpha$ en la red cristalina (Gunn, 1987; Karlsson, 1999). La precipitación de la nueva fase origina que la matriz quede enriquecida en níquel, elemento estabilizador de la $\gamma$, dando como resultado $\gamma_{2}$ con una composición química muy aproximada a la de la $\gamma$ generada a altas temperaturas (Gunn, 1987; Karlsson, 1999; Pohl et al., 2007).

La necesidad de evaluar por ultrasonido los daños en componentes de este tipo de acero se ha extendido de lo clásicamente detectable: dimensionamiento y defectología (convencional), a lo más complejo o no convencional (ASM, 1989; Rodríguez et al., 2011). Entre los ensayos no convencionales se encuentra la espectroscopía ultrasónica; ésta determina la dependencia de las propiedades y las características del material bajo estudio, con la frecuencia. Esta técnica ha sido empleada en la caracterización de materiales ya que cuando un pulso ultrasónico se propaga a través de un material, éste interactúa con sus componentes microestructurales (granos, inclusiones, grietas, etc.), y la misma puede ser cuantitativamente relacionada con sus tamaños y geometrías, así como con la longitud de la onda incidente. Esta interacción puede ser evaluada como un cambio en la distribución de frecuencias del eco transmitido (análisis espectral de eco), o de la señal de ruido (análisis espectral de ruido) (Rodríguez et al., 2011; Stella et al., 2009). No obstante, dada la complejidad que pueden presentar estas señales durante el análisis espectral y la limitación del análisis de Fourier en la resolución espectro-temporal, se ha considerado otro tipo de análisis no estacionario como lo es el análisis dinámico basado en la teoría del caos determinista (Strogatz, 1994).

La teoría del caos es una rama de las matemáticas y la física que estudia los sistemas dinámicos y en especial los sistemas complejos (Strogatz, 1994). Los sistemas dinámicos presentan el estudio de toda señal que evoluciona en el tiempo, según una ley física, presentando estados o fases dinámicas. Un tipo particular de dinámica lo constituyen las señales caóticas, las cuales se caracterizan por ser sensibles a las condiciones iniciales del sistema que las genera, lo que dificulta la predicción de su evolución a largo plazo (Strogatz, 1994; Suárez et al., 2007; Dumas et al., 1993).

En función de la evolución los sistemas dinámicos se puede clasificar en estables o inestables, periódicos, cuasi periódicos o caóticos. Se visualizan, en un espacio denominado espacio de fase dinámico, como vectores de campo que convergen, en el caso estable, o divergen, en el caso inestable, a trayectorias que delimitan la condición final del sistema. Estos límites dinámicos pueden ser puntuales, circulares en el caso periódico, toroidales en el caso cuasi periódico, o cambian en geometrías extrañas como en el caso caótico. De esta manera el espacio de fase es la representación ortogonal de los vectores de estado que agrupa el conjunto de las posibles respuestas (límites) del sistema. Dicho de otra manera, si tenemos las ecuaciones de estado que describen el sistema bajo estudio, las trayectorias de los estados en el espacio de fase ilustran todas las posibles soluciones de estas ecuaciones. Las geometrías que ofrecen estos vectores fueron clasificadas por Poincarè en 1890, como límites: punto crítico o singular, ciclos límites (órbitas), ciclos cuasi-periódicos (toro) y caos (atractor extraño). La Tabla 1, esquematiza las diferentes geometrías límites clasificadas por Poincarè. Tal y como se visualiza en esta tabla, el volumen del espacio hacia el cual las trayectorias son atraídas se denomina la cuenca de atracción y la variedad geométrica (punto, curva, superficie, etc.) asociada a esta cuenca se denomina atractor. Un sistema estable tiende a lo largo del tiempo a un punto, o a una órbita, según la dimensión del atractor. Un sistema inestable se escapa exponencialmente de la región de atracción en el transcurso del tiempo. Un sistema caótico combina ambos comportamientos: por un lado existe un atractor por el que el sistema se ve atraído, pero a la vez hay "fuerzas" que lo alejan de éste. De esa manera, el sistema permanece confinado en una zona del espacio de fase, sin tender a un atractor fijo. 
TABLA 1. Tipos de atractores

Límite
Punto Singular
Ciclo Límite
Cuasi periódico
Caos

La ventaja de las representaciones en el espacio de fases dinámico es que permite visualizar el comportamiento de las señales tanto en tiempo como en frecuencia, a través del concepto de atractor. Otra ventaja del análisis basado en la teoría del caos determinista es que abarca desde la potencialidad visual de su representación, hasta la estimación de parámetros discriminantes sobre los espacios de fase. Estos parámetros evalúan la complejidad, el comportamiento frecuencial, la entropía y la estabilidad dinámica del fenómeno estudiado, entre otros aspectos.

El análisis de los sistemas dinámicos requiere de la definición del proceso físico subyacente y del conocimiento de sus ecuaciones y variables de estado. No obstante, es un hecho que las leyes "dinámicas" que rigen la mayoría de los procesos en la realidad son desconocidas por lo que no es posible la construcción del espacio de fases. Ahora bien, como toda señal temporal es la proyección en una dimensión del comportamiento general del sistema del cual fue adquirido, entonces las señales en sí mismas contienen toda la información requerida para "recomponer" su dinámica. Esta conclusión fue planteada por primera vez por Takens, quien desarrolló un teorema que proporciona las condiciones bajo las cuales las trayectorias del espacio de fases pueden "reconstruirse" a partir de señales temporales discretas (Takens, 1981). Existen diferentes métodos de reconstrucción del espacio de fases. En el presente estudio se utiliza el método por retardos. Este método descrito por Takens (Takens, 1981) considera particularmente el análisis de señales temporales a tiempo discreto $\mathrm{X} t$, con $t=\left[T s, 2 T_{s} \ldots . . N T s\right]$, donde $N \in \mathrm{Z}$ y $T s$ es el periodo de muestreo. Es así como se generan $\mathrm{M}$ vectores contentivos de algunas versiones de la señal retardadas un factor $\tau$ (Ec. 1):

$$
X_{t}^{m}=\left|X_{t}, X_{t+\tau}, \ldots, X_{t+(M-1) \tau}\right|^{T}
$$

donde $\mathrm{M}$ es la dimensión de inmersión y es igual a $\mathrm{M} \geq 2[D]+1$ y $[D]$ indica el número entero más reducido, mayor que $D$. Por ejemplo, si considera$\operatorname{mos} T s=1$ y $\tau=1$ (Ec. 2 ):

$$
\begin{array}{ccccc} 
& x_{1} & x_{2} & \cdots & x_{N-(\mathrm{M}-1)} \\
X_{t}^{m} & x_{2} & x_{3} & \cdots & x_{N-(\mathrm{M}+2)} \\
& \vdots & \vdots & \ddots & \vdots \\
& x_{N-(\mathrm{M}-1)} & \cdots & & x_{N}
\end{array}
$$

Entre los parámetros obtenidos en el atractor se encuentran aquellos del tipo estático y los del tipo dinámico. Los del tipo estático describen la dimensión de la cuenca de atracción y la naturaleza del movimiento de la señal. Los parámetros dinámicos se relacionan con la evolución de la trayectoria, e incluyen la determinación de la estabilidad y la complejidad por medio del exponente de Lyapunov y de la entropía asociada a los cambios dispersivos de la dinámica de la señal (Proccacia, 1985). En este trabajo nos enfocaremos en los del tipo estático, específicamente en la estimación de la dimensión de información (D1) del atractor (Folland, 1989; Hidalgo, 2003; Fernández, 2005; Lyon et al., 1995).

La dimensión de información del atractor describe el comportamiento asintótico de un sistema; si la dimensión no es un valor entero, éste se denomina atractor extraño e implica la existencia de un comportamiento complicado y posiblemente caótico. Este tipo de información es útil en la descripción del sistema ya que es capaz de establecer el límite inferior de variables independientes requerido por la descripción de la dinámica del sistema (Hidalgo, 2003; Fernández, 2005).

Sea $\{x(t)\}$ la trayectoria de un sistema dinámico muestreado: $x(0), x(\Delta t), \ldots ., x(H \bullet \Delta t)$. Considere un espacio de fase de dimensión $D$, y una métrica basada en abiertos d-dimensiones que cubren las trayectorias del espacio de diámetro $\varepsilon$. Sea $M(\varepsilon)$ el número total de abiertos (celdas) necesarias para cubrir totalmente las trayectorias. La probabilidad de encontrar un punto de la cuenca de atracción en la i-ésima celda de diámetro será (Ec. 3):

$$
P i=\lim N \rightarrow \infty \frac{N_{i}}{N} \quad i=1, \ldots ; M(\varepsilon)
$$

donde $N i$ es el número de puntos de las trayectorias ubicadas en la cuenca de atracción que se encuentran dentro de la celda $\mathrm{i}$, y $N$ es el número 


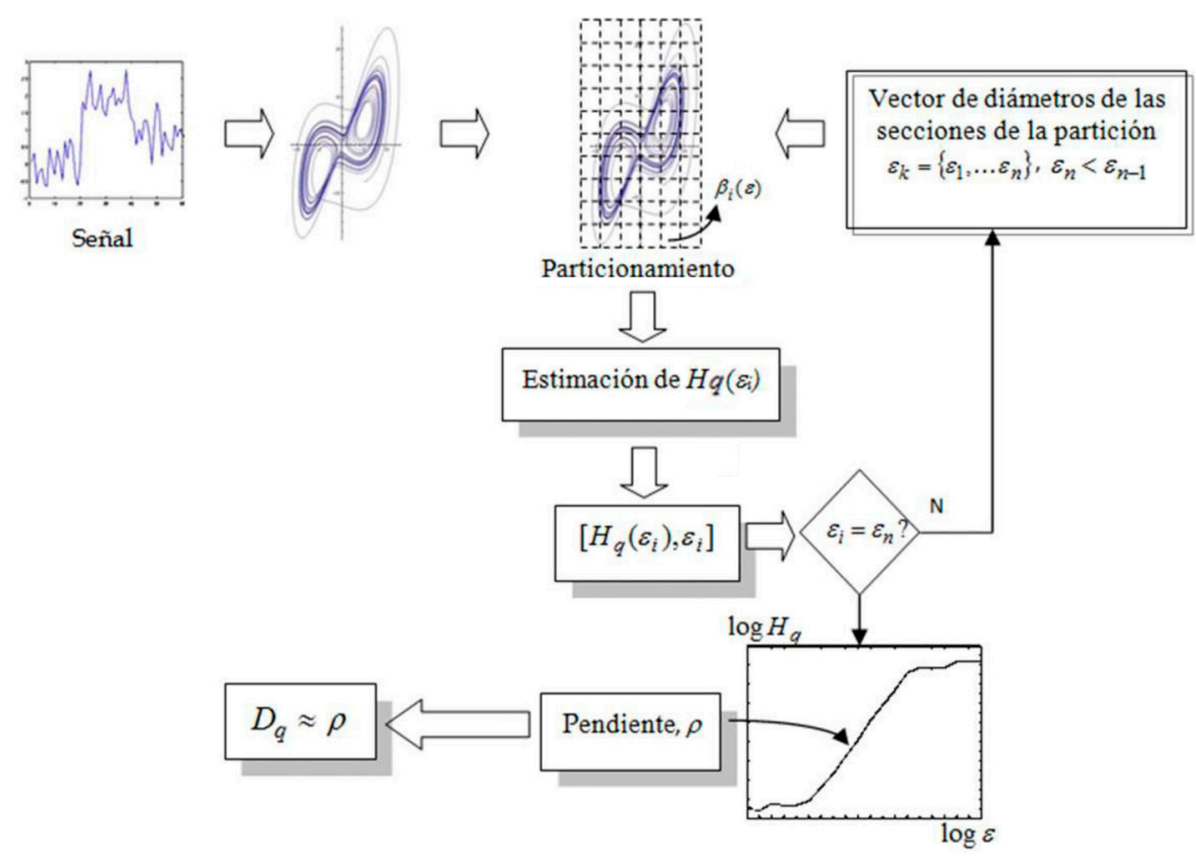

FIgURA 1. Algoritmo para la estimación de la dimensión del orden q de un atractor.

total de puntos del atractor (que pertenecen a la secuencia de muestreo). La dimensión de información caracteriza la estructura estática del atractor mediante la siguiente expresión (Ec. 4):

$$
D_{f}=\lim _{\mathcal{E} \rightarrow 0} \frac{1}{f-1} \frac{\log \sum_{i=0}^{M(\varepsilon)} p_{i}^{f}}{\log \varepsilon} f=0,1,
$$

Si se toma el límite para $f \rightarrow 1$, se obtiene la denominada $D 1$. La dimensión de información cuantifica el incremento de la ganancia de información a medida que $\varepsilon$ se aproxima a 0 (Folland, 1989). Experimentalmente, las dimensiones son estimadas por la aproximación sobre una curva $\log -\log$ que relaciona $H q$ y $\varepsilon$, donde $H q$ es la expresión de complejidad. Este algoritmo se desarrolla en la Figura 1.

A fin de caracterizar las señales por medio de los espacios de fases correspondientes a un acero dúplex tratado térmicamente, en este trabajo se cuantifican medidas estáticas del atractor, específicamente la $D I$ de cada una de las señales obtenidas mediante la evaluación ultrasónica de muestras de acero dúplex SAF 2205 durante la precipitación y disolución de la fase $\sigma$.

\section{MATERIALES Y MÉTODOS}

El material utilizado es un acero comercial SAF 2205 (UNS31803) en forma de barra. Las probetas de $20 \mathrm{~mm}$ de diámetro y $15 \mathrm{~mm}$ de espesor han sido tratadas térmicamente a $875^{\circ} \mathrm{C}$ y $950{ }^{\circ} \mathrm{C}$ y fueron preparadas metalográficamente de acuerdo a la norma ASTM E3-01 (ASTM, 2007) para ser caracterizadas por microscopía óptica. La evaluación ultrasónica por contacto directo se llevó a cabo considerando la cara frontal de las muestras en condición de pulido, haciendo incidir el haz ultrasónico en dirección paralela al eje de cada una de ellas. Por otra parte, se diseñó un sistema el cual consiste en dos placas paralelas de plexiglás, que permiten colocar la muestra y el palpador entre ellas, a fin de aplicar sobre el palpador una carga constante, para evitar las posibles variaciones en la amplitud de la señal. Con el fin de eliminar la capa de aire que se pudiera formar entre el transductor y la superficie de las probetas de ensayo, se utilizó glicerina como acoplante. El estudio se realizó utilizando un detector de fallas Krautkramer USN-60 y transductores de $6,35 \mathrm{~mm}$ e haz normal y frecuencias de 5 y 10 MHz. La atenuación en cada una de las muestras se determinó con base a la comparación de las amplitudes de los ecos de fondo, para lo cual se calculó el coeficiente de atenuación (Rodríguez et al., 2011).

Las señales fueron obtenidas por medio de un osciloscopio marca Tektronix modelo TDS 1002 de $60 \mathrm{MHz}$ y una longitud de registro de 2500 puntos por canal, con un periodo de muestreo de $4,0 \times 10^{-9}$ s y una frecuencia de muestreo equivalente a $2,5 \times 10^{8} \mathrm{~s}^{-1}$. Para la realización del análisis de las señales ultrasónicas se seleccionó la segunda señal proveniente del eco de fondo de la probeta, en Radio Frecuencia (RF), a fin de obviar los fenómenos del campo cercano del palpador. Se aisló la señal, de forma que apareciera únicamente ésta en la 


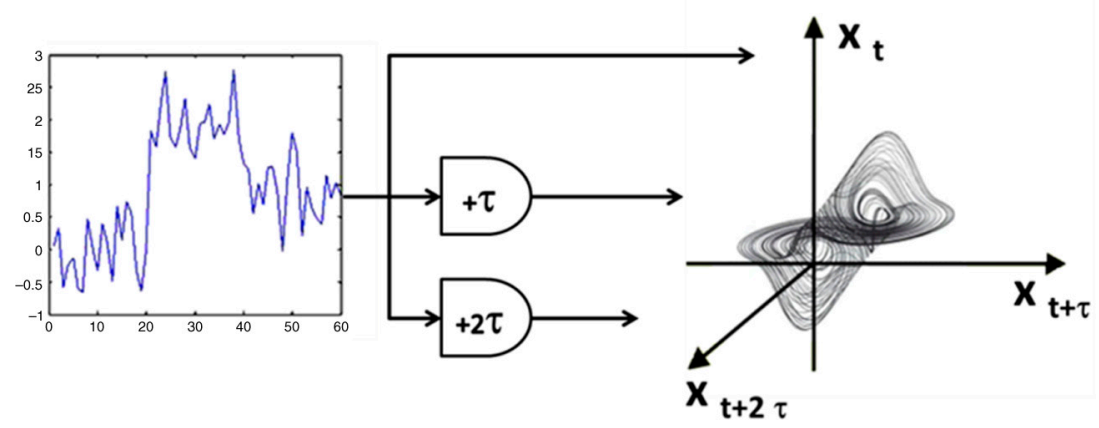

Figura 2. Reconstrucción del espacio de fases. Método por retardos (Suárez et al., 2007; Dumas et al., 1993).

pantalla y se procedió a almacenar los datos digitalizados de la señal. Para el análisis basado en la teoría del caos determinista y con los datos almacenados, se realizó la reconstrucción del espacio de fases dinámico de cada una de las señales correspondientes en cada una de las probetas evaluadas con base al método de retardos, con $M=2$ (Procaccia, 1985; Hidalgo, 2003). El procedimiento de reconstrucción consiste en asociar cada fila de la matriz $X t^{m}$ a un eje de coordenadas del espacio euclidiano de dimensión 2, tal como se muestra en la Figura 2. Cada punto de este espacio es un vector que contiene el conjunto de las amplitudes de una muestra de la señal y sus $N-m$ muestras vecinas o retardadas de un factor $\tau$.

Este método de reconstrucción requiere definir los parámetros del retraso $\tau$ y de la dimensión de inmersión $M$ (Takens, 1981; Fraser et al., 1983). El retardo fue determinado según los valores de información mutua por el procedimiento de Fraser (1983).

En cada espacio de fases, se compararon las amplitudes instantáneas de la señal (eje x) con las amplitudes retardadas un cierto número de muestras (eje y). Una vez reconstruido el espacio de fases, se determinó la D1. Esta dimensión nos indica la cantidad de información que se aporta al aumentar la resolución de las mediciones de los vectores de estado del sistema y los mismos se utilizaron como parámetros de caracterización en la evaluación de cada una de las probetas.

Por otro lado, a fin de visualizar gráficamente la relación existente entre la $D 1$ con las fracciones de cada una de las fases presentes en el acero y con el coeficiente de atenuación determinado para cada una de las frecuencias, se calculó el Coeficiente de Correlación de Pearson (CCP), que mide la relación lineal entre dos variables aleatorias y la cual es independiente de la escala de medida de las variables. Este factor varía en el intervalo $[-1,1]$, destacando que si está entre $0<\mathrm{r}<1$, existe una correlación positiva y una correlación negativa si está entre $-1<\mathrm{r}<0$. De ser igual a 1 o -1 existe una perfecta correlación positiva y negativa respectivamente, lo que indica una dependencia total entre las dos variables de forma directa o inversa y de ser igual a 0 no existe relación lineal entre las dos variables.

\section{RESULTADOS}

\subsection{Evolución microestructural}

La Figura 3 muestra la evolución microestructural obtenida por medio de microscopía óptica de cada una de las probetas tratadas a $875^{\circ} \mathrm{C}$ durante diferentes lapsos de tiempo, con el fin principal de promover una precipitación significativa de la fase sigma $(\sigma)$. En general, se aprecia a partir de 1800 segundos de tratamiento la precipitación de la fase $\sigma$ entre la interfase $\alpha / \gamma$ visualizándose con un alto contraste (zona muy oscura). En todos los casos, la fase $\sigma$ ha nucleado preferencialmente en la interfase $\alpha / \gamma$ y su crecimiento se ha expandido a partir de la fase $\alpha$ consumiéndola gradualmente (Rodríguez et al., 2011). Esta precipitación preferencial de la fase $\sigma$ a lo largo de la $\alpha$ ocurre por la alta velocidad de difusión de los elementos que conforman la fase $\alpha$ como el cromo y el molibdeno, motivada por la similitud entre la estructura tetragonal de la fase $\sigma$ y la estructura BCC de la $\alpha$ en la red cristalina. Por otro lado, la precipitación de esta nueva fase origina que la matriz quede enriquecida en níquel, elemento estabilizador de la $\gamma$, dando como resultado austenita secundaria $\left(\gamma_{2}\right)$ con una composición química muy aproximada a la de la $\gamma$ generada a altas temperaturas (Gunn, 1987; Rodríguez et al., 2011).

La Figura 4 muestra la evolución microestructural de las probetas durante los tratamientos de disolución de la fase $\sigma$ a $950{ }^{\circ} \mathrm{C}$, observándose una disminución de la fracción de la fase $\sigma$ (fase oscura), así como un incremento de la fase $\alpha$ (gris oscuro), a medida que aumenta el tiempo de tratamiento. Cabe destacar que los resultados obtenidos muestran una disminución de la fase $\sigma$, estabilizándose a medida que aumentamos el tiempo de tratamiento (Rodríguez et al., 2011).

Tal comportamiento obedece a que la temperatura mínima de disolución total de esta fase se encuentra alrededor de los $980{ }^{\circ} \mathrm{C}$ (Gunn, 1987; 


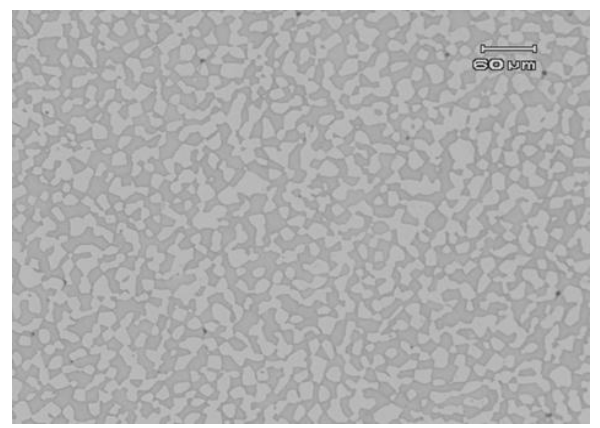

Condición original

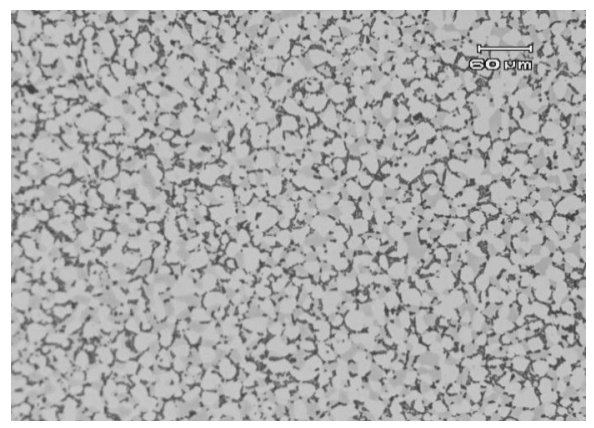

$21.600 \mathrm{~s}$

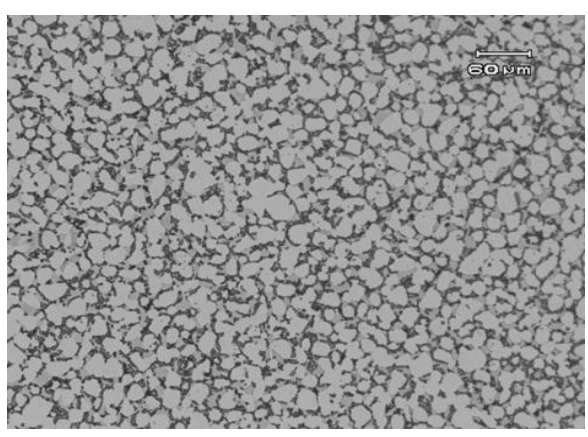

$7200 \mathrm{~s}$

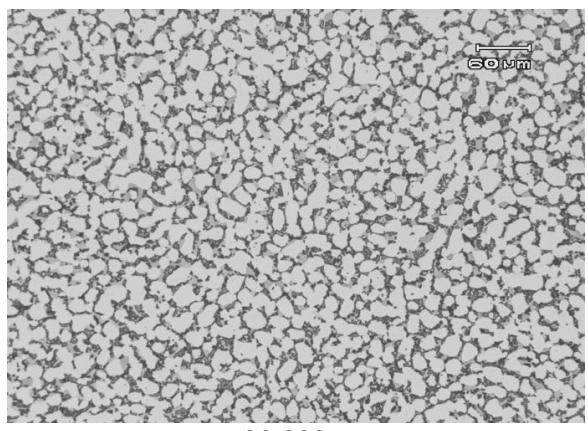

$36.000 \mathrm{~s}$

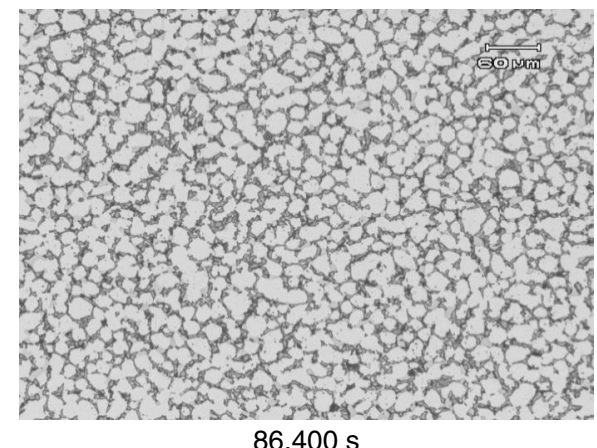

$86.400 \mathrm{~s}$

Figura 3. Evolución microestructural de las probetas tratadas a $875^{\circ} \mathrm{C}$ durante la precipitación de la fase sigma. Las zonas oscuras corresponden a la fase $\sigma$ y la clara la fase $\gamma$.

Rodríguez et al., 2011). La temperatura escogida en esta investigación permite la caracterización de muestras con disolución parcial de la fase $\sigma$, debido al interés que existe en evaluar la influencia de las fases presentes sobre el comportamiento de las señales (Rodríguez et al., 2011). La Tabla 2 muestra la fracción de área, determinada mediante metalografía óptica cuantitativa, para cada una de las probetas tratadas a 875 y $950^{\circ} \mathrm{C}$ (Rodríguez et al., 2011).

\subsection{Análisis basado en la dimensión de información}

\subsubsection{Tratamiento a $875^{\circ} \mathrm{C}$. formación de la fase $\sigma$}

Las Figuras 5 y 6 muestran los atractores en el espacio de fase de las señales obtenidas para algunas probetas que han sido tratadas térmicamente a $875^{\circ} \mathrm{C}$ por diferentes lapsos de tiempo determinados con los palpadores de 5 y $10 \mathrm{MHz}$, con el objetivo principal de hacer precipitar la fase $\sigma$. En cada una de ellas se visualiza la forma del atractor con cada uno de los palpadores y la misma varía entre el tipo cuasi periódico y caótico.

Por otra parte, en el centro de la cuenca de atracción de estos espacios se observa una alta probabilidad de estados, conforme a la ecuación (1) que corresponden a la presencia de ruido blanco en la señal. El ruido blanco es un ruido aleatorio que posee la misma densidad de potencia espectral a lo largo de toda la banda de frecuencias. Esto significa que la señal de ruido blanco contiene todas las frecuencias y todas ellas muestran estadísticamente la misma potencia espectral. En las Figuras 5 y 6 cada círculo o elipse corresponde a los ciclos asociados a una frecuencia. Llama la atención que para cada palpador a un tiempo de tratamiento dado, 


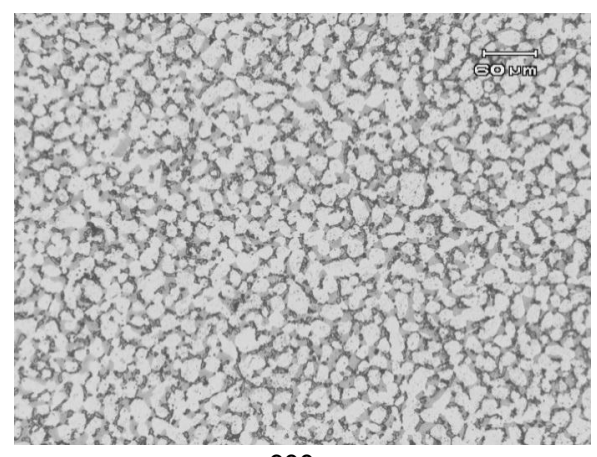

$600 \mathrm{~s}$

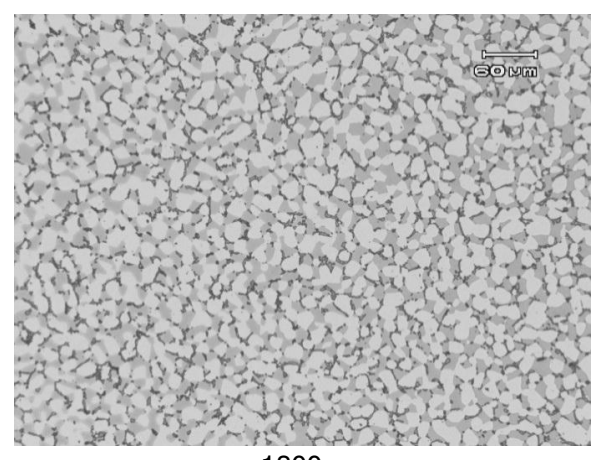

$1800 \mathrm{~s}$

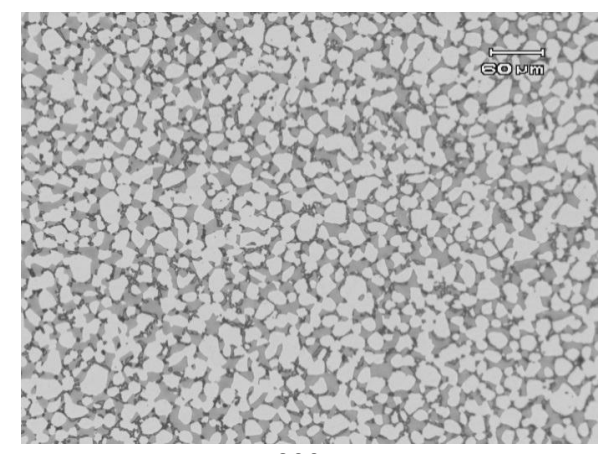

$900 \mathrm{~s}$

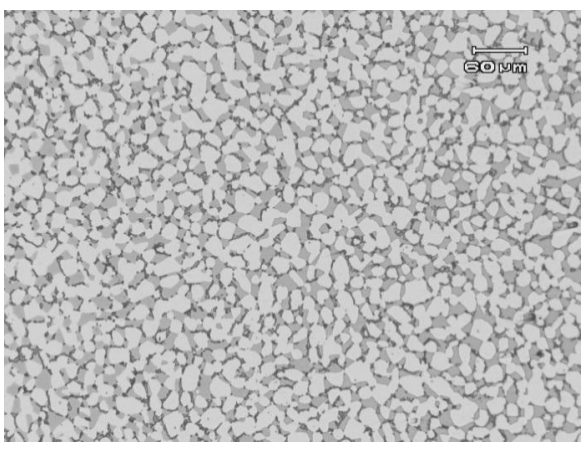

$3600 \mathrm{~s}$

FIGURA 4. Evolución microestructural de las probetas durante el tratamiento de disolución de la fase $\sigma$ a $950{ }^{\circ} \mathrm{C}$.

La zonas oscuras corresponden a la fase $\sigma$ y las claras la $\gamma$.

se exhibe un cambio de las órbitas mostrando una representación mucho más compleja a lo largo del tiempo. La complejidad visual infiere un incremento del número de variables dinámicas involucradas en el proceso y reflejadas en la naturaleza de sus trayectorias. Esto concuerda con los cambios que ocurren en el material principalmente por la variación de las fracciones de fases $\alpha, \gamma$ y $\sigma$. Cabe destacar que en el análisis de sistemas dinámicos, la complejidad es un término que se evalúa en función de parámetros específicos tales como la entropía, los cuales no fueron considerados en el marco de esta investigación. Sin embargo, la medida de la DI refleja el incremento de las variables de estado y por ende de la riqueza de la información de las señales. Es así como utilizando el palpador de $5 \mathrm{MHz}$, las mayores variaciones ocurren a 21.600 segundos y en el palpador de $10 \mathrm{MHz}$ se presentan a lo largo de todos los tiempos. Esto sugiere que el palpador de $10 \mathrm{MHz}$ permite una mejor visualización de los cambios que ocurren en el acero por efecto de los tratamientos térmicos.

Para cada uno de los atractores se cuantificó la Dl y dicho valor se utilizó como parámetro de caracterización dinámica en la evaluación de cada una de las probetas. Los resultados obtenidos se muestran

TABLA 2. Fracciones de las fases $\alpha, \gamma$ y $\sigma$ en función del tiempo de tratamiento

\begin{tabular}{|c|c|c|c|c|c|c|}
\hline \multirow[b]{2}{*}{ Tiempo (s) } & \multicolumn{3}{|c|}{$\begin{array}{c}\text { Tratamiento a } 875^{\circ} \mathrm{C} \\
\text { Formación de } \sigma \\
\end{array}$} & \multicolumn{3}{|c|}{$\begin{array}{c}\text { Tratamiento a } 950{ }^{\circ} \mathrm{C} \\
\text { Disolución de } \sigma\end{array}$} \\
\hline & $\alpha$ & $\sigma$ & $\gamma$ & $\alpha$ & $\sigma$ & $\gamma$ \\
\hline 0 & $0,48 \pm 0,01$ & $0 \pm 0$ & $0,52 \pm 0,01$ & $0,18 \pm 0,05$ & $0,14 \pm 0,02$ & $0,68 \pm 0,04$ \\
\hline 600 & - & - & - & $0,29 \pm 0,04$ & $0,11 \pm 0,02$ & $0,60 \pm 0,02$ \\
\hline 900 & - & - & - & $0,37 \pm 0,04$ & $0,09 \pm 0,02$ & $0,54 \pm 0,02$ \\
\hline 1800 & $0,28 \pm 0,04$ & $0,05 \pm 0,01$ & $0,67 \pm 0,02$ & $0,40 \pm 0,05$ & $0,08 \pm 0,02$ & $0,52 \pm 0,03$ \\
\hline 7200 & $0,21 \pm 0,02$ & $0,13 \pm 0,01$ & $0,66 \pm 0,01$ & - & - & - \\
\hline 21.600 & $0,17 \pm 0,01$ & $0,13 \pm 0,02$ & $0,70 \pm 0,02$ & - & - & - \\
\hline 36.000 & $0,15 \pm 0,01$ & $0,14 \pm 0,02$ & $0,71 \pm 0,01$ & - & - & - \\
\hline 86.400 & $0,15 \pm 0,02$ & $0,14 \pm 0,02$ & $0,71 \pm 0,02$ & - & - & - \\
\hline
\end{tabular}



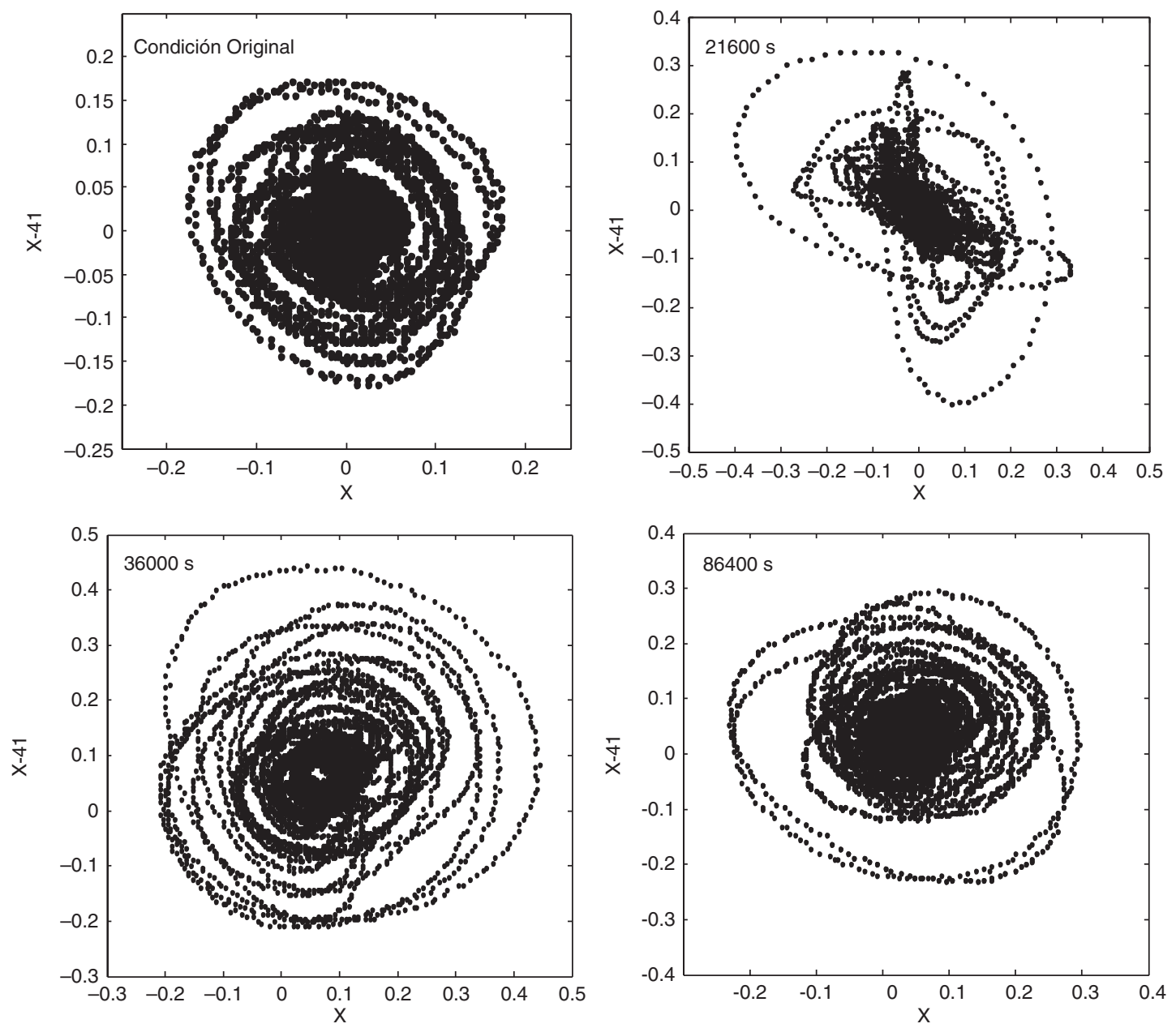

Figura 5. Atractores de la señal obtenida con el palpador de $5 \mathrm{MHz}$ en probetas tratadas térmicamente a $875^{\circ} \mathrm{C}$ en el que se promueve la fase $\sigma$. El eje x corresponde al vector de amplitudes de la señal ultrasónica mientras que el eje y contiene una versión retardada de dicho vector en 41 muestras.

en las Figuras $7 \mathrm{a}$ y $7 \mathrm{~b}$; en las mismas se aprecia la variación de $D 1$ con respecto al tiempo de tratamiento para cada uno de los palpadores utilizados. Los resultados obtenidos para cada una de las frecuencias muestran una dimensión no entera o fraccional-cuyo valor de dimensión es un número decimal - por cuanto los valores obtenidos están entre 1 y 2 aproximadamente; esto infiere la naturaleza caótica de la señal. En general, los valores para cada una de las frecuencias muestran variaciones de la $D I$ a medida que aumenta el tiempo de tratamiento.

Dicho comportamiento revela que la dinámica del sistema hasta 86.400 segundos de tratamiento muestra cambios significativos. No obstante, destaca que estos cambios sobre la dinámica son mucho más significativos entre los 1800 segundos y 36.000 segundos de tratamiento. Los valores más altos de la DI corresponden a un aumento en el número de las variables dinámicas implícitas en el fenómeno. Por ejemplo a 1800 segundos y utilizando el palpador de
$10 \mathrm{MHz}$ la $D 1$ es igual a 1,4 , tiempo en el cual las trayectorias presentan formas más complejas, en comparación al que se observa a 21.600 segundos cuya $D 1$ es igual 0,9 . Tales resultados se refieren a un aumento en el número de variables a 1800 segundos, lo que podría asociarse al comienzo de la precipitación de la fase $\sigma$. De esta forma el comportamiento de la $D 1$ sugiere que los mecanismos que actúan sobre la dinámica de la señal podrían estar asociados a las transformaciones que ocurren en el acero durante el lapso de tiempo en el que se produce la máxima precipitación de $\sigma$ y en el que varían significativamente las fracciones de la fase $\alpha$ y $\gamma$ por efecto de esta precipitación. En vista de estos cambios que ocurren en la dinámica del sistema, se graficaron para cada uno de los palpadores las fracciones de las fases presentes en cada una de las probetas durante el tratamiento a $875^{\circ} \mathrm{C}$ y de los parámetros acústicos a lo largo de los diferentes lapsos de tiempo con respecto a la dimensión de información $D 1$. 

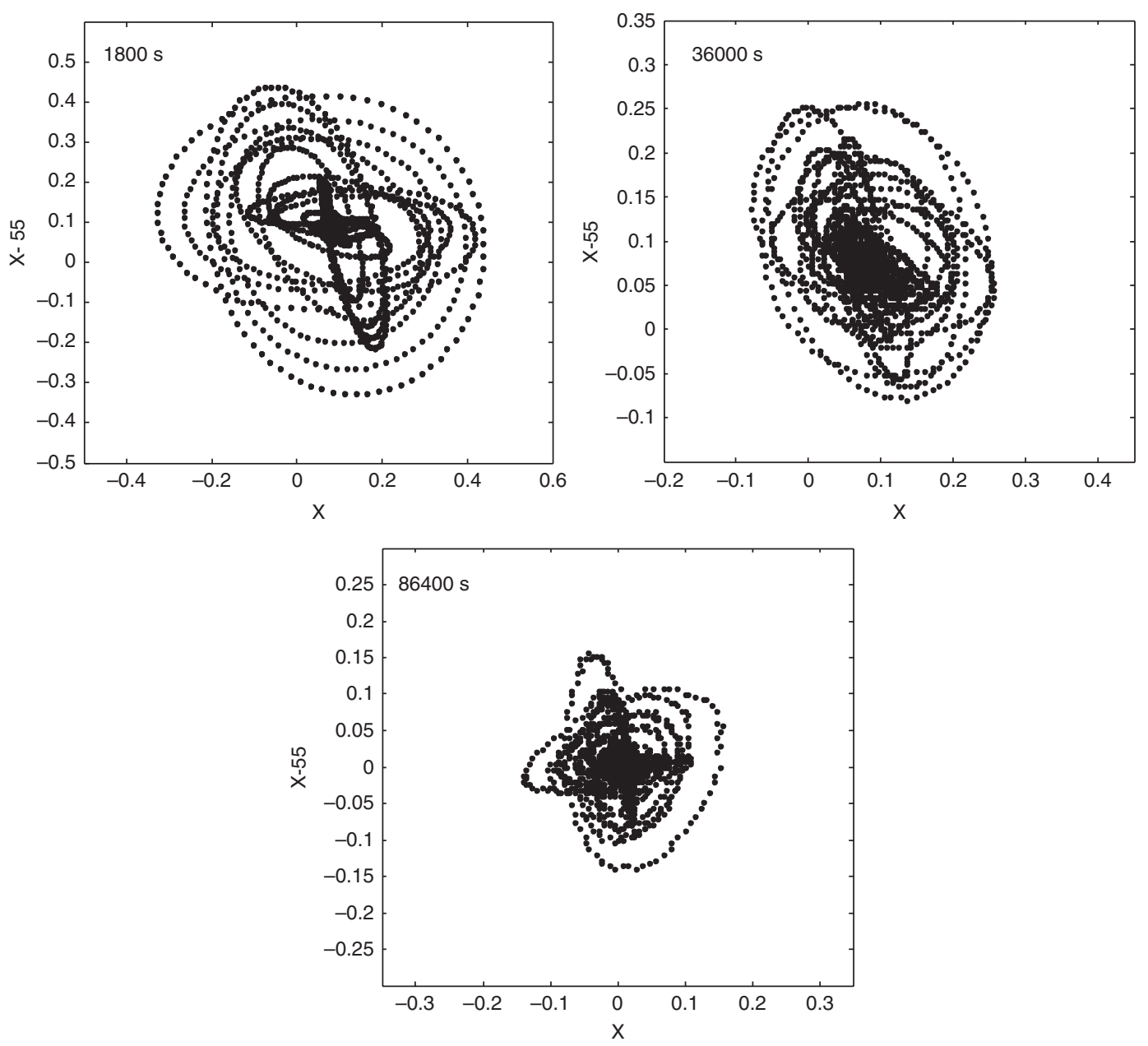

Figura 6. Atractores de la señal obtenida con el palpador de $10 \mathrm{MHz}$ de probetas tratadas térmicamente a $875{ }^{\circ} \mathrm{C}$ en el que se promueve la fase sigma. El eje x corresponde al vector de amplitudes de la señal ultrasónica mientras que el eje y contiene una versión retardada de dicho vector en 55 muestras.

Siguiendo un análisis comparativo de $D 1$ con respecto a las fracciones de fase $\alpha, \gamma$ y $\sigma$, la Figura $7 \mathrm{a}$ muestra los resultados de los valores correspondientes a la $D 1$ de cada una de las probetas ensayadas utilizando el palpador de $5 \mathrm{MHz}$ y de las fracciones de fase a medida que se aumenta el tiempo de tratamiento. Los resultados obtenidos muestran en general una dinámica cuasi periódica con más de una frecuencia principal del sistema, particularmente hasta 21.600 segundos, cuyos valores de $D 1$ tienden a mostrar cierta estabilidad a medida que aumenta el tiempo de tratamiento. Esta dinámica de la señal es el resultado de la contribución de todos los cambios que ocurren en el sistema particularmente hasta 21.600 segundos de tratamiento y la poca variación hasta 86.400 segundos de dicho tratamiento. Asimismo se aprecia que la misma sigue una tendencia inicial muy similar a las variaciones de la fase ferrítica y a partir de los 7200 segundos una tendencia que podría estar correlacionada con las variaciones de las fases $\gamma$ y $\sigma$. En cuanto al palpador de $10 \mathrm{MHz}$ (Fig. 7b), los resultados obtenidos indican nuevamente una dinámica compleja del sistema hasta 21.600 segundos de tratamiento mostrando muy poca variación a partir de 36.000 segundos de tratamiento. Asimismo al correlacionar el comportamiento de la $D 1$ con las fracciones de cada una de las fases $(\alpha, \gamma, \sigma)$, la gráfica muestra una tendencia inicial hasta 7200 segundos de tratamiento que se asemeja al comportamiento reflejado por la fracción de fase austenítica y seguidamente podría ser el resultado de una mayor contribución de la fase ferrítica. No obstante, el comportamiento observado es tan complejo que no se descarta la contribución del resto de las fases. La Tabla 3 muestra los valores obtenidos de la correlación entre las fracciones de fase y la D1 a través del CCP. Los resultados obtenidos indican una correlación positiva fuerte entre la fracción ferrítica y austenítica con la dimensión de información particularmente 
(a)

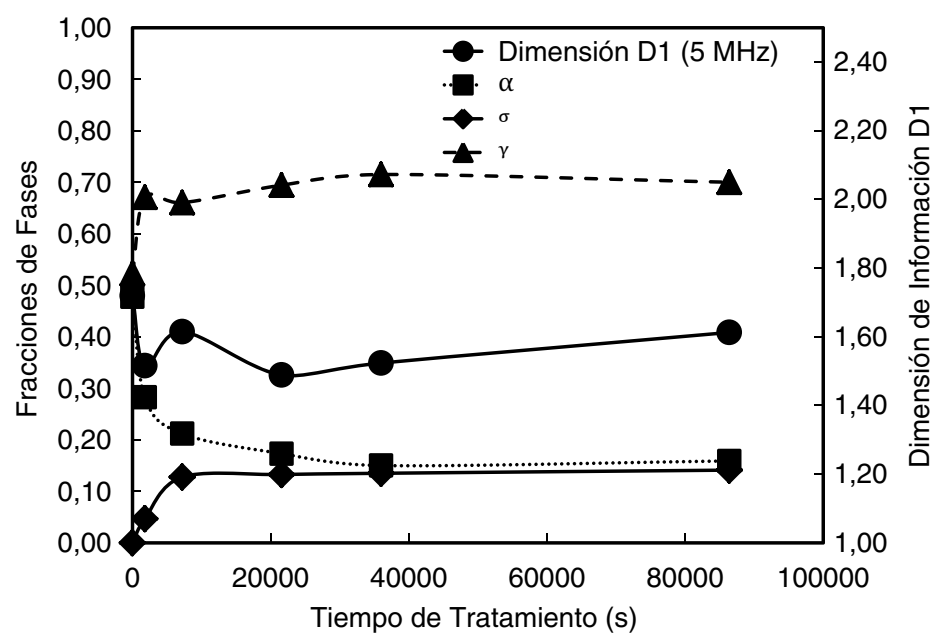

(b)

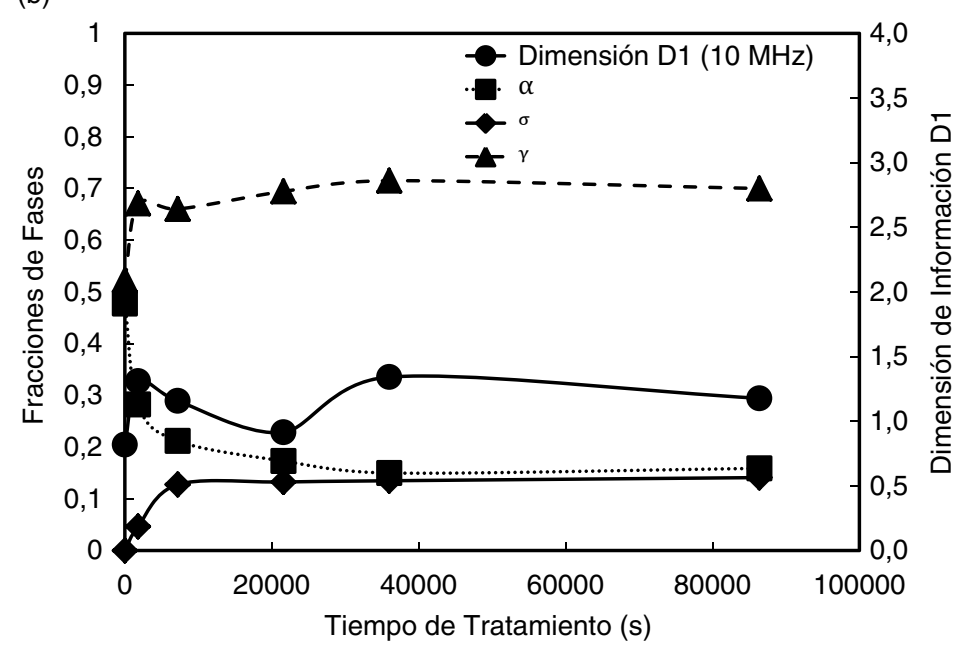

FiguRa 7. Dimensión de información de los atractores y fracciones de cada una de las fases presentes en cada una de las probetas evaluadas durante los tratamientos a $875^{\circ} \mathrm{C}$. (a) $5 \mathrm{MHz}$; (b) $10 \mathrm{MHz}$.

si se utilizan los palpadores de 5 y $10 \mathrm{MHz}$. Esto podría corroborar que la naturaleza de la señal se ve influenciada por estas fases y en particular con la fase $\gamma$, cuyas fracciones varían significativamente a medida que precipita la fase $\sigma$.

Ahora bien, considerando también que la $D 1$ del atractor puede representar también el flujo energético de la señal, se evaluó el comportamiento de la dimensión $D 1$ y del coeficiente de atenuación de las señales ultrasónicas.

Las Figuras $8 \mathrm{a}$ y $8 \mathrm{~b}$ muestran la $D 1$ versus el coeficiente de atenuación para cada una de las probetas evaluadas. La Figura 8a muestra que el comportamiento de $D 1$ utilizando el palpador de $5 \mathrm{MHz}$, se asemeja al reflejado por el coeficiente de atenuación particularmente entre los 1800 y 36.000 segundos

TABla 3. Coeficiente de correlación de Pearson (CCP) expresados como relación entre la dimensión de información $(D 1)$ y las fracciones de fase, en función de las temperaturas de tratamiento

\begin{tabular}{lcccc}
\hline Temperatura $\left({ }^{(} \mathbf{C}\right)$ & Frecuencia $(\mathbf{M H z})$ & $\mathbf{C C P}(\boldsymbol{D} 1 / \alpha)$ & $\mathbf{C C P}(\boldsymbol{D} 1 / \gamma)$ & $\mathbf{C C P}(\boldsymbol{D} 1 / \sigma)$ \\
\hline $875{ }^{\circ} \mathrm{C}$ & 5 & 0,7 & 0,8 & 0,5 \\
& 10 & 0,6 & 0,7 & 0,4 \\
$950{ }^{\circ} \mathrm{C}$ & 5 & $-0,5$ & 0,5 & 0,7 \\
& 10 & 0,5 & $-0,6$ & $-0,3$ \\
\hline
\end{tabular}




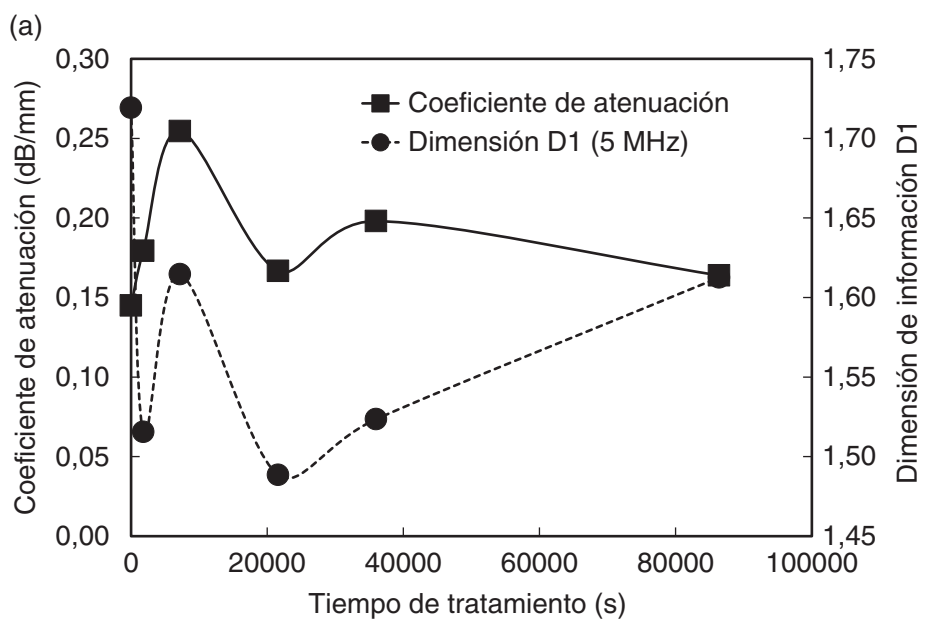

(b)

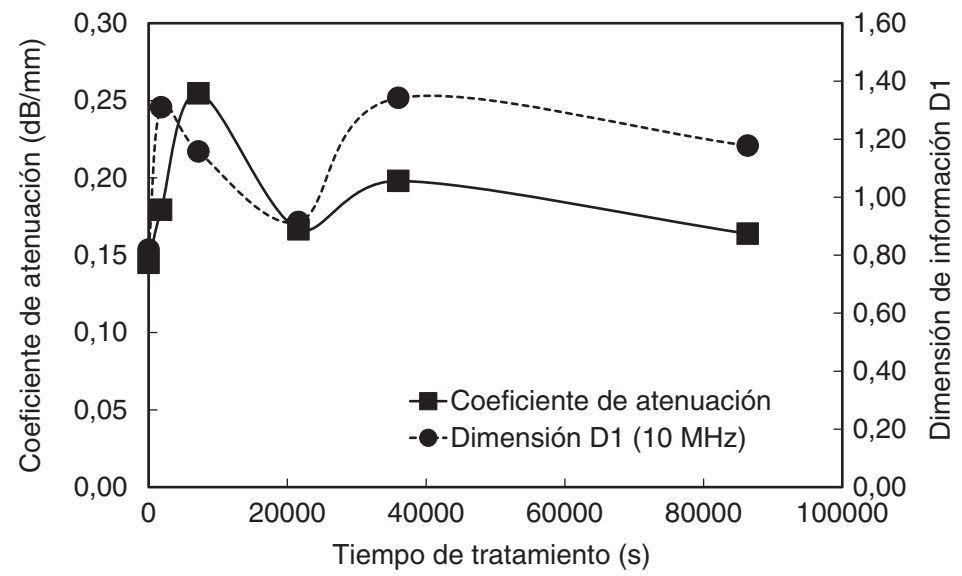

Figura 8. Dimensión de información de los atractores y coeficiente de atenuación durante los tratamientos a $875^{\circ} \mathrm{C}$. (a) $5 \mathrm{MHz}$; (b) $10 \mathrm{MHz}$.

de tratamiento, intervalo de tiempo en el cual ocurren cambios significativos particularmente por la precipitación de la fase $\sigma$. Por su parte, la Figura $8 \mathrm{~b}$ correspondiente al palpador de $10 \mathrm{MHz}$ presenta un comportamiento similar a la del coeficiente de atenuación a lo largo de todo el intervalo de tratamiento.

Este comportamiento sugiere que la dimensión de información de la señal $D 1$, calculada con el palpador de $10 \mathrm{MHz}$ podría correlacionarse con las pérdidas de energía que ocurren en el sistema a lo largo de todos los tiempos de tratamiento y que se

Tabla 4. Coeficiente de correlación de Pearson (CCP) expresados como relación entre la dimensión de información

(D1) y el coeficiente de atenuación, en función de las temperaturas de tratamiento

\begin{tabular}{lcc}
\hline & \multicolumn{2}{c}{ Frecuencia $(\mathbf{M H z})$} \\
\cline { 2 - 3 } Temperatura de tratamiento $\left({ }^{\circ} \mathbf{C}\right)$ & $\mathbf{5}$ & $\mathbf{1 0}$ \\
\hline 875 & $-0,2$ & 0,7 \\
950 & 0,5 & 0,5 \\
\hline
\end{tabular}

relacionan con los cambios microestructurales que se generan en cada una de las probetas a medida que se promueve la precipitación de la fase $\sigma$.

Los valores correspondientes al CCP entre el coeficiente de atenuación y la D1 (Tabla 4), indican una correlación positiva fuerte entre las dos variables utilizando el palpador de $10 \mathrm{MHz}$ y una poca o ninguna correlación para las dos variables utilizando el palpador de $5 \mathrm{MHz}$. Esto podría sugerirnos que el palpador de $10 \mathrm{MHz}$ es el más recomendado durante este tipo de evaluación principalmente por la relación positiva (lineal) que existe entre la dimensión de información y el coeficiente de atenuación.

\subsubsection{Tratamiento a $950^{\circ} \mathrm{C}$. Disolución de la fase $\sigma$}

Las Figuras 9 y 10 muestran los atractores en el espacio de fase de las señales obtenidas para algunas de las probetas que han sido tratadas térmicamente a $950{ }^{\circ} \mathrm{C}$ durante diferentes lapsos de tiempo, con el objetivo principal de disolver la fase $\sigma$, determinados con los palpadores de 5 y $10 \mathrm{MHz}$. 

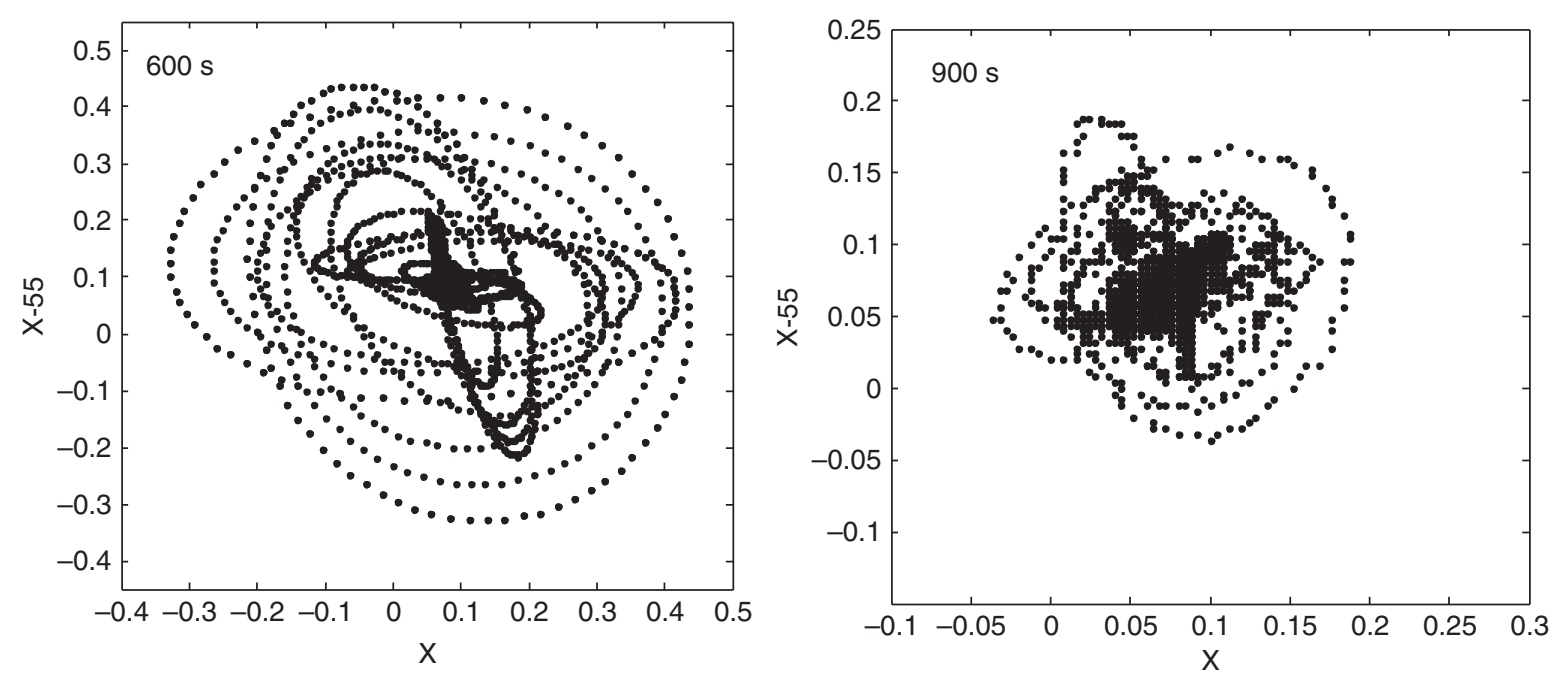

Figura 9. Atractores de la señal obtenida con el palpador de $5 \mathrm{MHz}$ en probetas tratadas térmicamente a $950{ }^{\circ} \mathrm{C}$, en el que se disuelve la fase $\sigma$. El eje x corresponde al vector de amplitudes de la señal ultrasónica mientras que el eje y contiene una versión retardada de dicho vector en 55 muestras.
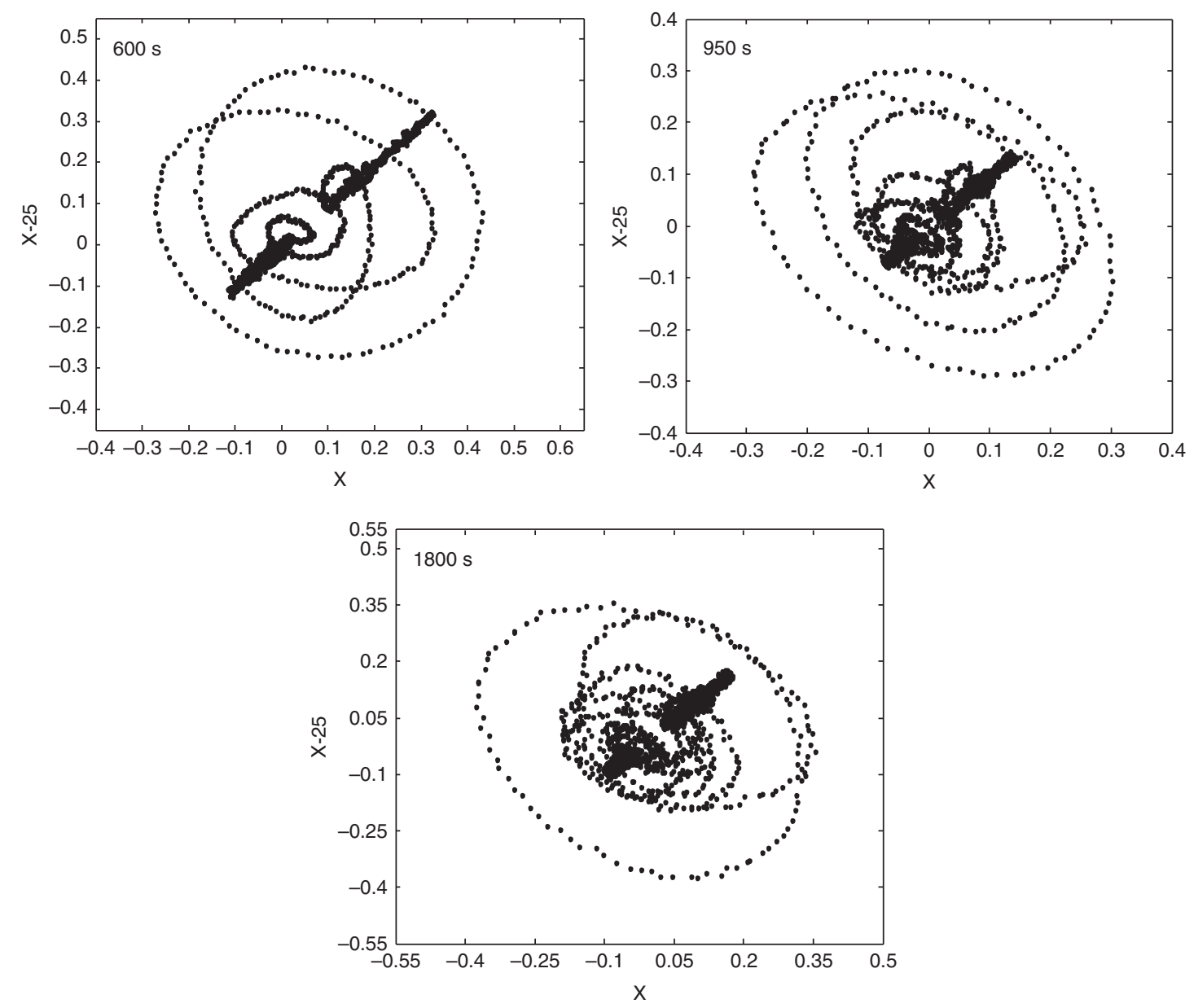

Figura 10. Atractores de la señal obtenida con el palpador de $10 \mathrm{MHz}$ en probetas tratadas térmicamente a $950{ }^{\circ} \mathrm{C}$, en el que se disuelve la fase sigma. El eje x corresponde al vector de amplitudes de la señal ultrasónica mientras que el eje y contiene una versión retardada de dicho vector en 25 muestras. 
(a)

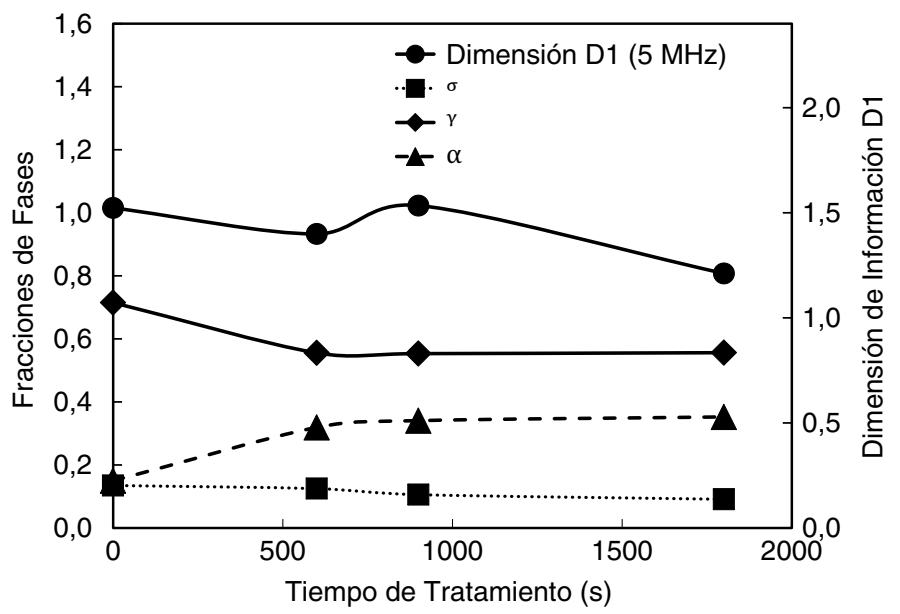

(b)

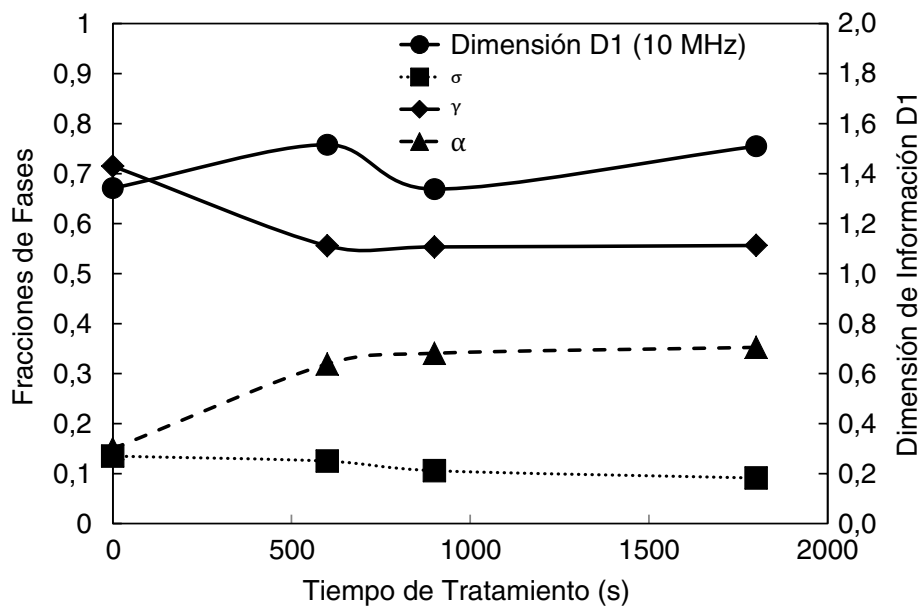

Figura 11. Dimensión de información de los atractores y fracciones de fase durante los tratamientos de disolución a $950^{\circ} \mathrm{C}$. (a) $5 \mathrm{MHz}$; (b) $10 \mathrm{MHz}$.

En cada una de ellas se distinguen atractores cuya forma es del tipo entre cuasi periódico y caótico, presentando zonas con una alta concentración de estados en la mayoría de los casos en el centro y que corresponde al llamado ruido blanco. Para cada uno de los atractores se cuantificó la $D 1$ y dicho valor se utilizó como parámetro de caracterización en la evaluación de cada una de las probetas. Los resultados obtenidos se muestran en la Figura 11, en donde se aprecia la variación de la $D 1$ con respecto al tiempo de tratamiento para cada uno de los palpadores utilizados. Los resultados obtenidos para cada una de las frecuencias muestran una dimensión no entera o fraccional - cuyo valor de dimensión es un número decimal - por cuanto los valores obtenidos están entre 1 y 2 aproximadamente.

En general los valores obtenidos para cada una de las frecuencias muestran una variación significativa de la dinámica de la señal a medida que aumenta el tiempo de tratamiento, particularmente con el palpador de $10 \mathrm{MHz}$ a 600 segundos y con el palpador de $5 \mathrm{MHz}$ a los 900 segundos. Dichos comportamientos podrían estar asociados a la efectividad que tienen cada uno de los palpadores en la evaluación y caracterización de las transformaciones que suceden en este acero y que se relacionan con la disolución de la fase $\sigma$ y reformación de la fase ferrítica.

En la Figura 11a se observan los resultados obtenidos con el palpador de $5 \mathrm{MHz}$. En la misma se aprecia que la dinámica de la señal varía significativamente a medida que aumenta el tiempo de tratamiento. Por otra parte, la gráfica muestra cierta tendencia inicial que se asemeja al comportamiento reflejado por la fracción de la fase $\sigma$, lo que podría indicar una mayor contribución de esta fase en la dinámica de la señal, coincidiendo con el intervalo de tiempo en que ocurre su mayor disolución, de un 15 a un $8 \%$. Sin embargo, a partir de los 600 segundos no se aprecia correlación alguna de manera 
(a)

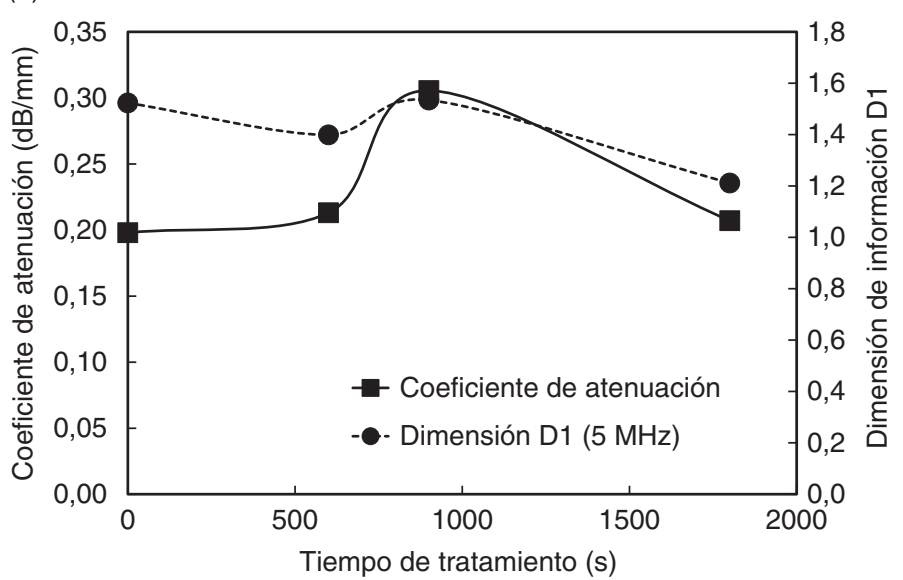

(b)

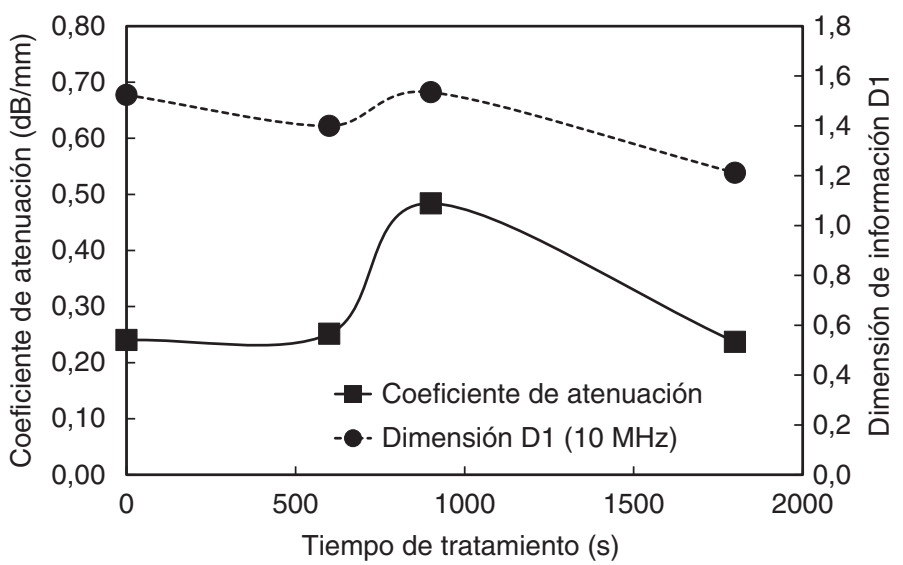

Figura 12. Dimensión de información de los atractores y coeficiente de atenuación durante los tratamientos de disolución a $950^{\circ} \mathrm{C}$. (a) $5 \mathrm{MHz}$; (b) $10 \mathrm{MHz}$.

individual con cada una de las fases presentes. Los resultados obtenidos con el palpador de $10 \mathrm{MHz}$ (Fig. 11b) muestran una variación de la dinámica de la señal particularmente a cortos tiempos de tratamiento y que coincide con el intervalo de tiempo en el que ocurre la mayor disolución de $\sigma$. Asimismo se observa cierta tendencia inicial que se asemeja al comportamiento de la fase ferrítica, lo que sugiere una mayor contribución de esta fase en la dinámica de la señal.

Los valores del CCP indican que el palpador de 5 $\mathrm{MHz}$, muestra una fuerte correlación positiva entre la $D 1$ y la fase $\sigma$ y un poco más débil con la fase $\gamma$ (Tabla 3). Este comportamiento con la fase $\sigma$ podría estar asociado a la alta inestabilidad de esta fase en este intervalo de temperatura. Por otro lado, se aprecia una cierta correlación de forma negativa con la fracción ferrítica. En cuanto a los resultados obtenidos con el palpador de $10 \mathrm{MHz}$, éstos muestran una correlación débil con pendiente negativa con la fase $\gamma$ y del tipo positiva con la fracción de la fase ferrítica, mostrando muy poca dependencia lineal con las fracciones de la fase $\sigma$.
La Figura 12 visualiza el comportamiento de $D 1$ y el coeficiente de atenuación con los palpadores de 5 y $10 \mathrm{MHz}$. En general las gráficas muestran que las dos curvas presentan un comportamiento similar a medida que aumenta el tiempo de tratamiento.

Por su parte, los valores del CCP (Tabla 4) indican una correlación positiva aunque débil entre las dos variables utilizando los palpadores de 5 y 10 MHz. Esto sugiere que utilizando estos dos palpadores el comportamiento de la dimensión de la señal podría asociarse de forma positiva aunque débil al comportamiento energético de la señal durante los tratamientos de disolución de la fase $\sigma$.

\section{CONCLUSIONES}

La evaluación microestructural del acero inoxidable dúplex estudiado muestra que a medida que aumenta el tiempo de tratamiento a $875^{\circ} \mathrm{C}$, se aprecia un incremento significativo de la fase sigma precipitada en las interfases ferrita-austenita. Por su parte, los tratamientos de disolución a $950{ }^{\circ} \mathrm{C}$ revelan una disminución de la fracción de la fase $\sigma$. 
Para los palpadores de 5 y $10 \mathrm{MHz}$ los espacios de fase obtenidos en cada una de las probetas presentan la forma de un atractor entre los tipo cuasi periódico y caótico. Por otra parte, en el centro de estos espacios de fases se observa una zona con una alta concentración de estados, agrupados sin una geometría identificable. Este fenómeno corresponde a la dinámica aleatoria del ruido blanco de la señal.

En general los resultados obtenidos para cada uno de los tratamientos térmicos de envejecimiento a $875{ }^{\circ} \mathrm{C}$ y de disolución a $950{ }^{\circ} \mathrm{C}$ muestran una dimensión no entera o fraccional cuyo valor de dimensión está entre 1 y 2 aproximadamente y que hace pensar en la naturaleza caótica de las señales.

Los resultados de la dimensión de información reflejan un comportamiento de la dinámica de la señal que se asemeja a las mediciones del coeficiente de atenuación, lo que sugiere una relación directa de $D 1$ con las pérdidas de la energía que ocurren en el sistema, particularmente en lapsos de tiempo en los que tienen lugar las variaciones más significativas por efecto de los cambios microestructurales. De esta forma, los resultados obtenidos indican una correlación positiva fuerte, es decir, casi una total dependencia de forma positiva entre las dos variables utilizando el palpador de $10 \mathrm{MHz}$ durante los tratamientos a $875^{\circ} \mathrm{C}$, donde se promueve la fase sigma y una correlación positiva aunque débil entre las dos variables utilizando los palpadores de 5 y 10 $\mathrm{MHz}$ durante los tratamientos de disolución.

En cuanto a la correlación entre la dimensión de información y las fracciones de fases $\alpha, \gamma$ y $\sigma$, los resultados obtenidos durante el tratamiento a $875^{\circ} \mathrm{C}$ muestran que la naturaleza de la señal se ve influenciada por las fases $\alpha$ y $\gamma$, particularmente con la fase $\gamma$, cuyas fracciones varían significativamente a medida que precipita la fase $\sigma$. En cuanto a los tratamientos de disolución a $950{ }^{\circ} \mathrm{C}$, los resultados obtenidos con el palpador de $5 \mathrm{MHz}$ muestran una fuerte correlación positiva entre la $D 1$ y la fase $\sigma$ y un poco más débil con la fase $\gamma$.

La dinámica de la señal es el resultado de la contribución de todos los cambios que ocurren en el sistema, permitiendo de esta forma destacar los intervalos de tiempo en los que suceden la mayor parte de las transformaciones en el material por efecto de la temperatura.

\section{AGRADECIMIENTOS}

Los autores agradecen al Decanato de Estudios de Postgrado de la Universidad Simón Bolívar, al Fondo Nacional de Ciencia, Tecnología e Investigación
(FONACIT) de Venezuela y a Sandvik de Venezuela por suministrar, en calidad de donación, el material bajo estudio.

\section{REFERENCIAS}

ASM International Handbook Committee Metals Handbook, 1989. ASM Handbook Nondestructive Evaluation and Quality Control. Ninth Edition, 17, Ohio, USA.

ASTM Standard E3-01, 2007. Standard Guide for Preparation of metallographic specimens ASTM International, West Conshohocken, USA.

Dumas, H.S., Laskar, J. 1993. Global dynamics and long-time stability in Hamiltonian systems via numerical frequency analysis. Phys. Rev. Lett. 70 (20), 2975-2979. http://dx.doi. org/10.1103/PhysRevLett.70.2975.

Fernández, J.G. 2005. Caracterización y filtrado de señales inmersas en ruido caótico y estocástico. Tesis de Doctorado, Facultad de Ingeniería, Universidad Nacional del Mar de Plata, 30-42.

Folland, G.B. 1989. Harmonic analysis in phase space, Princeton University Press.

Fraser, A.M., Swinney, H.L. 1986. Independent coordinates for strange attractors from mutual information. Phys. Rev. A 33 (2), 1134-1140. http://dx.doi.org/10.1103/PhysRevA.33.1134.

Gunn, R. 1987. Duplex stainless steels. Microstructure, properties and applications. Woodhead Publishing Ltd., 15-55.

Hidalgo, R. 2003. Sincronización y Caos en Sistemas Electrónicos no Lineales y sus aplicaciones a las Comunicaciones. Tesis de Doctorado, Universidad Nacional del Mar de Plata. Facultad de Ingeniería, Dpto. de Electrónica, Argentina.

Karlsson, L. 1999. Intermetallic Phase Precipitation in Duplex Stainless Steels and Weld. Welding Research Council Bulletin 438, 1-20.

Lyon, R.H., DeJon, R.G., Heckl, M. 1995. Theory and application of statistical energy analysis. J. Acoust. Soc. Am. 98 (6), 3021-3021. http://dx.doi.org/10.1121/1.413875.

Pohl, M., Storz, O., Glogowski, T. 2007. Effect of intermetallic precipitations on the properties of duplex stainless steel. Mater. Charact. 58, 65-71. http://dx.doi.org/10.1016/j. matchar.2006.03.015.

Procaccia, I. 1985. The static and dynamic invariants that characterize chaos and the relations between them in theory and experiments. Phys. Scripta T9, 40-46. http://dx.doi. org/10.1088/0031-8949/1985/T9/005.

Rodríguez, E., Stella, J., Kryzanowskyj, A., Amorer, L., Mateo, A. 2011. Caracterización de la respuesta ultrasónica de un acero dúplex 2205 durante la disolución de la fase sigma. Rev. LatinAm. Metal. Mat. 1, 49-60. http://www.rlmm.org/ ojs/index.php/rlmm/article/view/141.

Rodríguez, E., Stella, J., Ruiz, A., Fargas, G., Mateo, A. 2011. Characterization of microstructural changes in a duplex stainless steel using spectral analysis and conventional ultrasonic techniques. Materials Testing 53 (9), 564-571. http://dx.doi.org/10.3139/120.110264.

Stella, J., Cerezo, J., Rodríguez, E. 2009. Characterization of the sensitization degree in the AISI 304 stainless steel using spectral analysis and conventional ultrasonic techniques. NDT \& E Int. 42 (2), 267-274. http://dx.doi.org/10.1016/j. ndteint.2008.11.005.

Strogatz, S.H. 1994. Nonlinear dynamics and chaos. Perseus Books Publishing L.L.C. Cambridge, Massachusetts.

Suárez, K., Najim, M., González, C. 2007. Dimension analysis in atrial fibrillation's spontaneous termination. CLAIB 2007, IFMB Proceedings 18, 154-157.

Takens, F. 1981. Detecting strange attractors in turbulence en Dynamical Systems and Turbulence. Ed. Rand y Young, Springer, Berlin. 